\title{
Evaluation Model Construction and Simulation Research on Tourist Diversion Strategy in Ecotourism Scenery Sport
}

\author{
Shaojiang Lin, Yuguo Yuan, Weimin Zheng, Peiyu Ren and Maozhu Jin* \\ Business School, Sichuan University, Chengdu 610064, China
}

Received: 6 Jan. 2013, Revised: 9 May. 2013, Accepted: 10 May. 2013

Published online: 1 Nov. 2013

\begin{abstract}
Due to unbalanced distribution of tourists during peak time, tourists are likely to highly flock in certain areas of scenery spots if imperfect organization and management. For this reason, there may be crowds or even trample accidents in addition to unrecoverable damages for the ecological environment in such areas. Therefore, the valid solution is to formulate scientific tourist diversion strategy, reasonably adjust the tour routes, and realize the balanced distribution of tourists over space and time. The prerequisite and base of the research is to establish scientific rational evaluation indicators for diversion strategy. At present, the common indicators include the Variance Model reflecting the balance of load rate for each spot in the scenic region and the Gini-Simpson Index (G-S Index) showing the dispersion degrees of tourists among the scenery spots. However, these indexes highlight the opinions of scenery region managers but ignore the tourism utility during the travel. Such indexes can be used to evaluate the diversion strategy only from a macro view but hardly facilitates shaping any scientific valid suggestions for plan improvement. So this thesis established the evaluation model of tourism utility function (TUF) based on the spot congestion degree and compared the simulation effects of three diversion strategies respectively with three evaluation models, including Variance, G-S Index and TUF. It is found from the comparison results that TUF can better the overall evaluation of the diversion strategies and reveal the relevant inherent problems so as to provide reasonable and suggestions for scientific management on the scenery spot.
\end{abstract}

Keywords: Tourist diversion strategy, evaluation model, simulation, environment protection, ecotourism.

\section{Introduction}

Tourism development is an effective way for economic development and environment protection at the same time [1], and it not only means admission income to scenic region but also motivates the development of other related industries [2]. Nevertheless, greatly pushing forward the regional economy the rapid development of tourism also causes tremendous adverse effects on the ecological environment and social culture in the scenic region. During the peak time, the tourists highly crowd around in short period, leading to worse contradiction between economic development and environment protection in the scenic spots. For instance of Jiuzhaigou with the optimum capacity per day of 180,000 , this place accommodated over 40,000 tourists during the peak time of Golden Week around Chinese National Day in 2011 and great adverse results are made on the local ecological environment, mainly covering trample on the greening, damages to the soil structure, landscape losses, and consequential water loss and soil erosion; meanwhile, the nutrients affects the water clarity of the fringe water and the water quality, and further speeds up the process of lake alga bloom and bogginess etc. after directly flowing to the lake [3]. In addition, the unbalanced tourist distribution is likely to result in queuing crowd during the peak time and even trample accident, for example, for Beijing Miyun Festival Lantern Exhibition on February 5, 2004, the tourists flocked in hot scenery spots and the consequential super-high tourist density and crowd finally led to safety accidents after continuous squeezing with the toll of 37 deceased and 24 injured [4]. Therefore, the valid solution is to formulate scientific tourist diversion strategy, reasonably adjust the tour routes, and realize the balanced distribution of tourists over space and time. For this purpose, the coordination of tourists is commonly taken as planning or scheduling issue, and the relevant

\footnotetext{
*Corresponding author e-mail: jinmaozhu@ scu.edu.cn
} 
researchers quantify the travel routes from two dimensions $\mathrm{C}$ time and space in order to identify the combination optimization model for tourist coordination [5].

Based on the formation causes of tourism peak time in the scenic region, Feng Gang [5], Qiu Yanqing [6], Liu Zhusheng [7] and other researchers combined information technology like RFID and management theory and studied the space-time diversion and navigation management model related to tourists in the peak time. They made scientific diversion design and established such management model of the tourists in the scenic region from the two dimensions of time and space in order to reduce the instantaneous load of certain scenery spot and balance the space-time distribution. So they can realize several targets such as increase of tourist capacity, promotion of environment protection level, and better tourist satisfaction.

For the researches mentioned above, the variance of load rate in each spot or area is generally taken as only index for diversion strategy evaluation while the minimum variance is selected as objective function. And variance refers to the parameter or statistical quantity used to measure the degree of variance among all variables. It is used to evaluate the issues in many fields. However, this model has no fluctuation or deviation direction and the deviations of different directions lead to different or even opposite effects. Moreover, this evaluation model only appraises the overall space-time balance degree of certain scenic region from macro view. So Shi Jiangang introduced Gini-Simpson Index to evaluate the crowd or disperse features [8] of tourists in each scenery spot with quantitative analysis. However, the above two evaluation models both highlight the views of manager but ignore the specific feelings of tourists in the entire sight-seeing. To this end, this thesis established the evaluation model of tourism utility function (TUF) based on the spot congestion degree and compared the simulation effects of three diversion strategies respectively with three evaluation models, including Variance, G-S index and TUF. It is found from the comparison results that TUF can better the overall evaluation of the diversion strategies and reveal the relevant inherent problems so as to provide reasonable and suggestions for scientific management on the scenery spot.

\section{Establishment of Mathematical Model}

\subsection{Definitions of parameters}

$A$ : Set of scenery spots; $B$ : Set of tourists; $c_{j}$ : Natural capacity of the scenery sport $j ; t_{j}$ : Average stay duration of tourists in the scenery spot; $s_{j k}$ : Operation time between the spots $j$ and $h ; t_{i j}$ : Arrival time for tourist $i$ to the spot $j ; \triangle \tau_{i j}$ : Actual stay duration of tourist $i$ in the spot $j$. At the time $t$, the defined variable:

$$
x_{i j t}=\left\{\begin{array}{c}
1, \text { Tourist } i \text { has sight-seeing in the spot } j \\
\text { at the time } t\left(\text { when } t-s \cdot t_{i j} \leq t_{j}\right) \\
0, \text { Otherwise }
\end{array}\right.
$$

So the number of tourists in the spot $j$ at the time $t$ is $N_{j}^{t}=\sum_{i \in B} x_{i j t}$.

The load rate of the spot $j$ at the time $t$ :

$$
R_{j}=\frac{N_{j}^{t}}{c_{j}}=\frac{\sum_{i \in B} x_{i j t}}{c_{j}},(j=1,2, \cdots, n), R_{j} \geq 0 .
$$

The conventional load rate of scenery spot is gained from the ratio of load to capacity, among which the capacity is identified according to the factors such as natural and ecological environment, but for the capacity of tourism environment, such calculation model is almost static and the consequential capacity is only an ideal static result. In fact, the capacity is a dynamic system [9] continuously changing as the consumer behavior changes, tourism destination develops and the tourism period extends. Besides the above-mentioned factors, the capacity of the scenery spot is also related to the stay duration of tourist in the spot. Thus, Ge Peng and others proposed the concept of space-time load rate [10], while the definition is as follows:

$$
R_{j}^{s}=\frac{\sum_{i \in B} x_{i j t}}{\frac{c_{j}}{t_{j}}}=\frac{t_{j} \cdot \sum_{i \in B} x_{i j t}}{c_{j}},
$$

where $R_{j}^{s} \geq 0 ; j=1,2, \cdots, n$.

\subsection{Objective function}

The important goal pursued by the spot management is to realize the balanced space-time distribution of tourists in the spot and further ensure the orderly movement of tourists. And the goal means the increase of tourist capacity without damages to ecological environment. The tourists ask for utility value obtained as much as possible during the sight-seeing. Therefore, the researchers shall perform comprehensive evaluation from spot management and tourists when they evaluate the peak-time tourist diversion strategies. Fortunately, the balanced space-time distribution of tourists can also reduce the waiting time and uncomfortable feelings due to crowds and better the tour satisfaction. So the two goals have certain positive correlation to a great extent.

\subsubsection{Goal of spot balance degree}

Because of great differences among all spot capacities, the distribution balance does not represent distribution balance of tourist number but the balance of tourist load rate. Ideally if the load rate of each spot is balanced at any time $t$, the load rates of all spots is considered to reach balance over the entire period composite consisting of several continuous $t s$. And the spot space-time balance 
degree is converted to the model establishment of realizing all spots' load rates under optimum balance at certain time. Meanwhile, variance is used to measure the deviation degree between data and its average. Therefore, the Literature 7 described the establishment of Variance Model as evaluation index of spot tourist diversion strategy, shown as Eq.(3). However, this objective function only stresses the overall load balance of the scenic region, that is, the balance of space load rate in the scenic region, but ignores the time load balance of each scenery spot. So Shi Jiangang introduced Gini-Simpson Index to evaluate the features of gathering or dispersing process for each spot with quantitative analysis.

$$
Z=\operatorname{Min} \frac{1}{n-1} \sum_{j \in A}\left(R_{j}-\bar{R}\right)^{2}
$$

Where $R_{j}$ represents the rate load of the spot $j$ and $\bar{R}$ represents average load rate of all spots, shown as Eq.(4):

$$
\bar{R}=\frac{1}{n} \sum_{j \in A} R_{j}
$$

G-S Index is short for Gini-Simpson Index and the arrival rate for each admission period is taken as random variable $\xi$, so the relevant Gini-Simpson Index is recorded as $G-S(\xi)$ :

$$
G-S(\xi)=1-\sum_{t=1}^{T} p_{t}^{2}
$$

Where $T$ means the number of stages classified, and $p_{t}$ represents the admission ratio for each stage. Under the same classification conditions, larger G-S Index indicates higher dispersion degree of its distribution and the system closer to even distribution status.

\subsubsection{Tourism utility function}

Utility means the consumer's satisfaction [11] during the use of consumables while the tourism utility refers to comprehensive proceeds obtained from one trip. Belonging to non-monetary material gains, such proceeds mean the intrinsic emotional expression and psychological feelings such as happiness and excitement during the travel, sight-seeing and experience process of tourists [12]. There are many factors on tourism utility [13] in general grouped into costs and actual feelings. And the later usually depends on individual preferences of tourists, while it is hard to be observed but reflected indirectly by other indexes because such feelings belong to subjective psychological state. This thesis applied certain simplification during establishment of tourism utility function and the author believed that the time and costs consumed by the tourist during the travel of the same spot have no obvious differences, so the tourism utility is only related to the actual feelings of the tourists. The author also ignores the differences among the individuals but the effects of congestion degree on the tourism utility when he took account of the actual feelings.

At present, the tourists' psychological capabilities are covered generally by the consideration scope during the calculation of spot capacity; therefore, the utility value is the maximum when the number of tourists is no more than the relevant capacity, but the marginal utility will show certain descending law if the number exceeds the capacity. Moreover, the descending speed may gradually slow down as the number of tourists increases, that is:

$$
\left|\frac{d u}{d N}\right|_{N=N_{j}^{t_{1}}}>\left|\frac{d u}{d N}\right|_{N=N_{j}^{t_{2}}},\left(N_{j}^{t_{2}}>N_{j}^{t_{1}}>c_{j}\right)
$$

The tourism capacity is established based on the congestion degree according to the above analysis, and the tourism utility of tourist $I$ in the spot $j$ is shown as Eq.(7):

$$
u_{i j}= \begin{cases}1, & \bar{N}_{j}^{t_{i j} \rightarrow t_{i j}+\triangle \tau_{i j}} \\ \frac{1}{\exp \left[\left(\frac{\bar{N}_{j}^{t_{i j} \rightarrow t_{i j}+\Delta \tau_{i j}}-c_{j}}{c_{j}}\right)\right]}, & \text { else }\end{cases}
$$

Where $\bar{N}_{j}^{t_{i j} \rightarrow t_{i j}+\triangle \tau_{i j}}$ represents average number of tourists in the spot $j$ from the arrival time $t_{i j}$ to the departure time $t_{i j}+\triangle \tau_{i j}$ for the tourist $i$ :

$$
\begin{aligned}
& \bar{N}_{j}^{t_{i j} \rightarrow t_{i j}+\triangle \tau_{i j}} \\
& =\sum_{t=t_{i j}}^{t_{i j}+\triangle \tau_{i j}} \frac{N_{j}^{t}}{\triangle t_{i j}}
\end{aligned}
$$

Correspondingly, the utility value of the spot $\mathrm{j}$ in such period is the sum of all tourists' utility values in this spot during such period, shown as Eq.(9):

$$
U_{j}=\sum_{t=t i j}^{t_{i j}+\triangle \tau_{i j}} \sum_{i \in B} u_{i j} \cdot x_{i j t}
$$

To describe this utility model, for the spot $\mathrm{j}$ the capacity is $c_{j}=100$ while the marginal utility value $u_{i j}$ of the tourist and the tourism utility $U_{j}$ are shown as Figure 1. In the figure, $U_{j}$ reaches maximum as $\bar{N}_{j}^{t_{i j} \rightarrow t_{i j}+\triangle \tau_{i j}}=c_{j}$.

\section{Establishment of Simulation Model}

\subsection{Basic Data of Simulation}

Tourists show independence during the travel, so individual tourist takes certain personalized and random tourism route. In general, most tourists' routes show certain regularity, for an instance, Feng Gang designed 16 main tourism routes according to the statistics from Jiuzhaigou over years and measured the stay duration and 


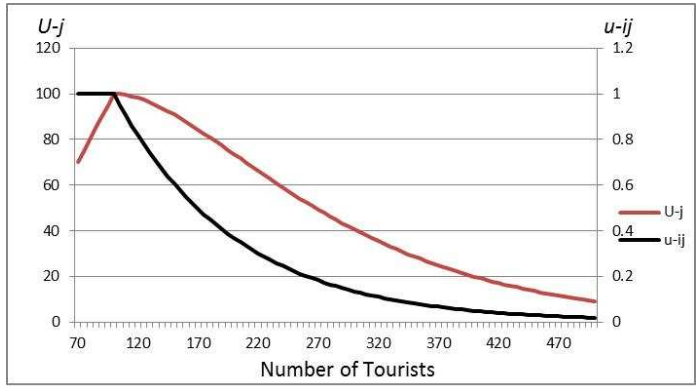

Fig. 1: Relationship Diagram of Tourist Space-Time Utility and Number of Tourists.

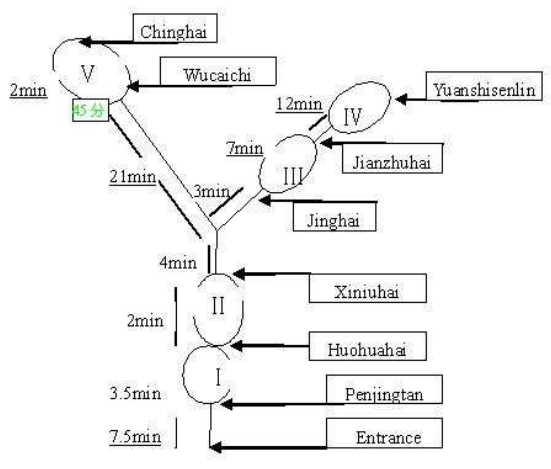

Fig. 2: Traffic Diagram of Jiuzhaigou.

diversion time scale [14] for each spot. The simulation model in this thesis applied the basic data and tourism routes collected by Feng's research, and the traffic diagram of Jiuzhaigou spots is shown in Figure 2 while the capabilities and average stay durations for 8 spots are listed in Table 1.

Table 1: Capacity and Tourist Stay Duration

\begin{tabular}{c|c|c|c|c}
\hline NO. & Spot & Capacity & $\begin{array}{r}\text { Average } \\
\text { stay duration }\end{array}$ & $\begin{array}{r}\text { Standard } \\
\text { deviation }\end{array}$ \\
\hline 1 & Penjingtan & 144 & 10 & 1 \\
2 & Huohuahai & 239 & 20 & 2 \\
3 & Xiniuhai & 950 & 30 & 4 \\
4 & Jinghai & 350 & 15 & 2 \\
5 & Jianzhuhai & 414 & 20 & 2 \\
6 & Yuanshishenlin & 480 & 30 & 3 \\
7 & Wucaichi & 60 & 15 & 2 \\
8 & Changhai & 268 & 30 & 3 \\
\hline
\end{tabular}

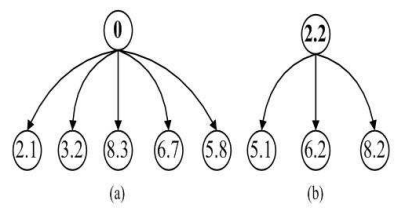

Fig. 3: Primary and Stabilized Decision Points of Scenic Region

\subsection{Description of simulation plan}

To reduce the simulation complexity, Ge Peng and the others [15] split the scenic region into unilateral network and categorized the nodes in the region into decision points and diversion points while the former is further grouped into primary and stationary decision points according to different positions. Shown as Figure 3, (a) represents a primary point where the tourist decides the goal node at the entrance of scenic region 0 , while $(b)$ is a stabilized decision point.

\subsubsection{Average strategy}

Average Strategy means the same shunt probabilities of tourists at the primary or stabilized decision points going to the downstream diversion nodes. Take Figure 3 for instance, the probabilities are the same for tourist allocated at the primary decision point 0 to the nodes 2.1 , $3.2,8.3,6.7$ and 5.8 while the relevant values are all 0.2 ; and at the stabilized decision point 2.2, the probabilities are all $\frac{1}{3}$ for tourists allocated to the nodes 5.1, 6.2 and 8.2.

\subsubsection{Average \& pace-time strategy (AST)}

For AST, as the tourist arrives at the primary decision point, the researcher applies average strategy while he can compare the space-time load rates of the downstream nodes available from this decision point and guide the tourists to the nodes with minimum rates for the tourists at the stabilized decision point. Also taking Figure 3 for instance, the probabilities are all 0.2 for tourists at primary decision points allocated to the 5 downstream nodes while the management shall compare the space-time load rates of the 3 downstream nodes for stabilized decision point and select the node with minimum rate from nodes 5.1, 6.2 and 8.2 as the goal node of the tourist.

\subsubsection{Logit strategy}

Average strategy as one of static diversion cannot make real-time adjustment according to the changes of spot load rates in the scenic region while AST as one of real-time 
diversion is likely to cause Matthew Effect. So Li Jin and others allocated the tourists to different routes with Logit Model and avoid the Mathew Effect to a great extent [16]. For the advantages of Logit Model, it can perfectly reflect the crowd and random factors during the route selection so as to realize the even space-time distribution of tourists and ensure the reasonable configuration of resources. This model is applicable for complex traffic and large network.

The diversion probability of the follow-up target spots $k\left(k \in T_{i j}\right)$ selected by the tourist $i$ at stabilized decision point $j$ :

$$
p(i, j, k)=\frac{\operatorname{Exp}\left[-\sigma \cdot \frac{R_{k}^{s}}{\bar{R}^{s}}\right]}{\sum_{k \in T_{i j}} \operatorname{Exp}\left[-\sigma \cdot \frac{R_{k}^{s}}{\bar{R}^{s}}\right]}
$$

Where $R_{k}^{s}$ is the space-time load rate of the spot $k ; \bar{R}^{s}$ is the average space-time load rate of the spot $k\left(k \in T_{i j}\right) ; T_{i j}$ is the set of follow-up target spots $k\left(k \in T_{i j}\right)$ selected by the tourist $i$ at stabilized decision point $j ; \sigma$ is nonnegative number, and when $\sigma=+\infty$, Logit Model is equal to the diversion based on space-time load rate, but as $\sigma=0$, it is average diversion, that is, the diversion rates of all nodes are the same. Obviously, the diversion probability is 0 for the node $k\left(k \notin T_{i j}\right)$.

Also taking Figure 3 for instance, Eq.(10) is used to calculate the diversion probabilities of $p(i, 2.2,5.1)$, $p(i, 2.2,6.2)$ and $p(i, 2.2,8.2)$ respectively as those for the tourists at the three scenery spots according to the space-time load rates of the three nodes 5.1, 6.2 and 8.2.

\subsection{Analysis of simulation results}

From Table 2 and Figure 4, the value of average strategy is better than those of the other two plans at the index of average spot load rate and the maximum and average variances of average strategy are bigger than those based on Logit Model, while the fluctuation figure of the former's variance shapes a relatively concentrated wave crest of the peak traffic time from 12 to 15 o'clock in one day. The big variance within this period highlights the load unbalance among all spots of the scenic region. The variance wave crests are relatively dispersed for AST and Logit strategies as the average and maximum of Logit Strategy is the lowest among these three strategies. Therefore, the Logit Model is doubtless the best at this index.

Table 2: Three Strategies' Comparison of load Rates and Variances

\begin{tabular}{c|c|c|c}
\hline Strategy & Average & AST & Logit \\
\hline Average-Load-Rate & 2.438 & 2.448 & 2.461 \\
Max-Variance & 45.4 & 68.35 & 43.71 \\
Average-Variance & 8.797 & 10.157 & 8.573 \\
\hline
\end{tabular}

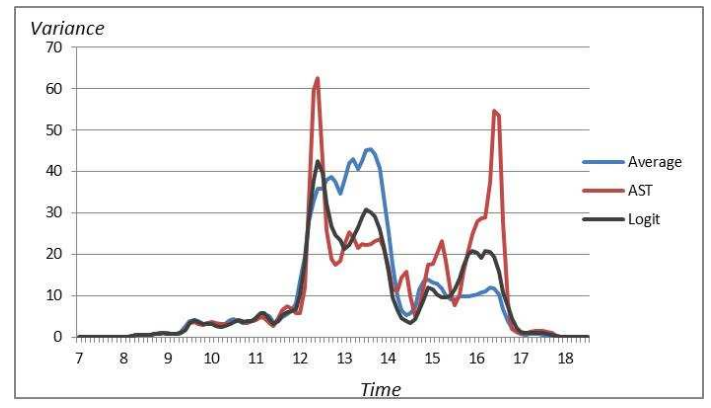

Fig. 4: Three Strategies Comparison of load Rate Variances

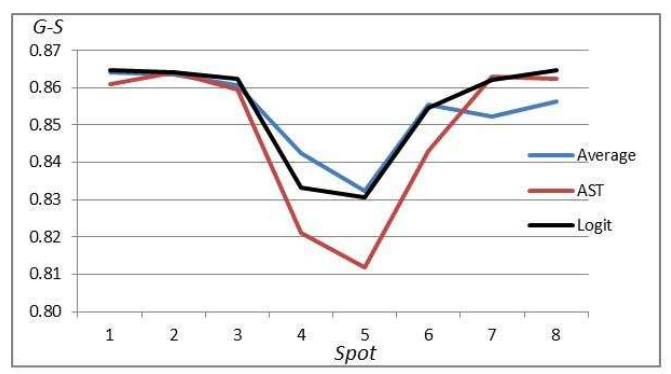

Fig. 5: G-S Index Comparison of Each Scenery Spot under the Three Strategies

It is supposed in the simulation that each tourist only visits every spot once, so each scenery spot will accommodate the same number of tourists with the value equal to the total tourists entering the region on that very day. Obviously, the arrival time of tourists to the same spot are dispersed approximately and the time distribution of tourists is much more balanced for the same spot. So this strategy can abate the damages of tourism actions to the ecological environment, reduce the management complexity, increase the capability and promote the tourism satisfaction. Taking the spot 2 for instance, Table 3 shows the arrival numbers of tourists over each period under these three strategies. Each hour is taken as a period from 7 to 19 o'clock when all tourists all depart from the region. And the arrival numbers of tourists are ideally the same over all periods and G-S Index $1-\sum_{t=1}^{1} 2=0.9167$ shows the best dispersion degree and most balanced distribution for tourists arriving at each spot. It is obvious that larger G-S Index value and better dispersion degree with more balanced distribution. So the larger value also leads to better local ecological environment protection, regional management and tourism satisfaction of tourists.

Table 3 and Figure 5 list the $G-S$ Index values and comparisons of each spot under three diversion strategies. It is easily found from the comparison that the $G-S$ Index values of each scenery point are different under different strategies and no strategy $\alpha$ stays within $G-S_{j}^{\alpha} \geq G-$ 
Table 3: Spot 2 Arrival Numbers of Tourists over Each Period

\begin{tabular}{cccc}
\hline Time & Average & AST & Logit \\
\hline $07: 00 \rightarrow 08: 00$ & 167 & 167 & 167 \\
$08: 00 \rightarrow 09: 00$ & 1410 & 1421 & 1410 \\
$09: 00 \rightarrow 10: 00$ & 2523 & 2482 & 2566 \\
$10: 00 \rightarrow 11: 00$ & 2417 & 2459 & 2457 \\
$11: 00 \rightarrow 12: 00$ & 1393 & 1327 & 1372 \\
$12: 00 \rightarrow 13: 00$ & 910 & 624 & 684 \\
$13: 00 \rightarrow 14: 00$ & 2782 & 2268 & 2065 \\
$14: 00 \rightarrow 15: 00$ & 4429 & 4286 & 4131 \\
$15: 00 \rightarrow 16: 00$ & 3086 & 3575 & 3798 \\
$16: 00 \rightarrow 17: 00$ & 830 & 1270 & 1248 \\
$17: 00 \rightarrow 18: 00$ & 53 & 121 & 101 \\
$17: 00 \rightarrow 18: 00$ & 0 & 0 & 0 \\
\hline
\end{tabular}

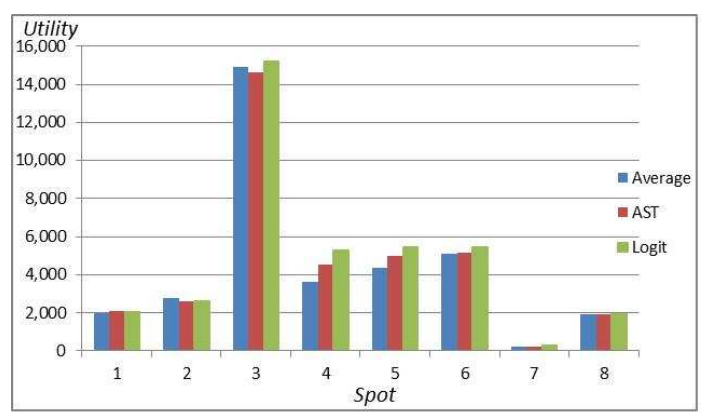

Fig. 6: G-S Index Comparison of Each Scenery Spot under the Three Strategies

$S_{j}^{\beta}(j \in A ; \alpha \neq \beta)$, so Logit Strategy is better than the other two from the comparison of $G-S$ Index.

Table 4: G-S Index Comparison of Each Scenery Spot under the Three Strategies

\begin{tabular}{cccc}
\hline O. & Average & AST & Logit \\
\hline 1 & 0.8642 & 0.8608 & 0.8646 \\
2 & 0.8636 & 0.8642 & 0.8642 \\
3 & 0.8607 & 0.8594 & 0.8624 \\
4 & 0.8425 & 0.8211 & 0.8211 \\
5 & 0.8323 & 0.8118 & 0.8306 \\
6 & 0.8556 & 0.8431 & 0.8545 \\
7 & 0.8524 & 0.8629 & 0.8620 \\
8 & 0.8564 & 0.8625 & 0.8648 \\
total & 0.8648 & 0.8648 & 6.8362 \\
\hline
\end{tabular}

From horizontal comparison of this tourism utility, there is no diversion strategy $\alpha$ satisfying Utility $_{j}^{\alpha} \geq$ Utility $_{j}^{\beta} ;(j \in A ; \alpha \neq \beta)$; However, it is found that Logit Strategy is the best among the three strategies at the utility value of several spots $j=3,4, \cdots, 8$.
Table 5: Spot Utility Value Comparisons of Diversion Strategies

\begin{tabular}{cccc}
\hline Spot & Average & AST & Logit \\
\hline 1 & 1991.57 & 2087.19 & 2050.2 \\
2 & 2794.26 & 2620.14 & 2611.98 \\
3 & 14924.71 & 14640.62 & 15235.35 \\
4 & 3596.99 & 4519.75 & 5282.24 \\
5 & 4350.63 & 4971.82 & 5459.79 \\
6 & 5107.4 & 5158.6 & 5478.8 \\
7 & 204.23 & 216.1 & 294.95 \\
8 & 1925.06 & 1897.33 & 1927.75 \\
total & 34894.85 & 34894.85 & 38341.06 \\
\hline
\end{tabular}

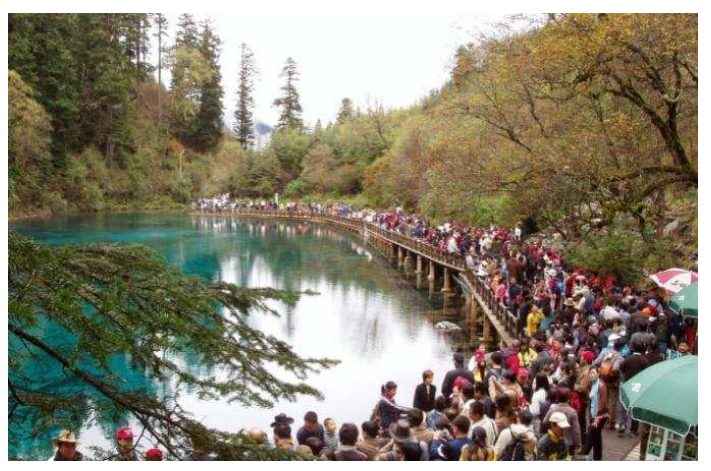

Fig. 7: Actual Congestion of Spot 7 during Peak Time

Table 6: Comprehensive Comparisons of Three Diversion Strategies Effects

\begin{tabular}{cccc}
\hline Index & Average & AST & Logit \\
\hline Max-Variance & 45.4 & 68.35 & 43.71 \\
Average-Variance & 8.797 & 10.157 & 8.573 \\
Total G-S Index & 6.8276 & 6.7859 & 6.8362 \\
Total Utility & 34894.85 & 34894.85 & 38341.06 \\
\hline
\end{tabular}

Table 6 shows the comprehensive comparisons of three diversion strategies' effects. It is indicated from the above-mentioned index analysis that Logit Strategy is better than the other two. From this view, the three evaluation strategies have the consistent appraisal process. However, the evaluation models of average strategy and AST can be only used for macro analysis of the diversion strategies but fail to reveal the inherent problems of each strategy, and TUF Evaluation Model can compensate these two models.

From longitude comparison of all spot utility values in Figure 6 and Table 5, though the tourism utility values differs for each spot under different diversion strategies, the utility value of the spot 7 is far less than the other spots' values. Therefore, it is necessary to further analyze this spot. 


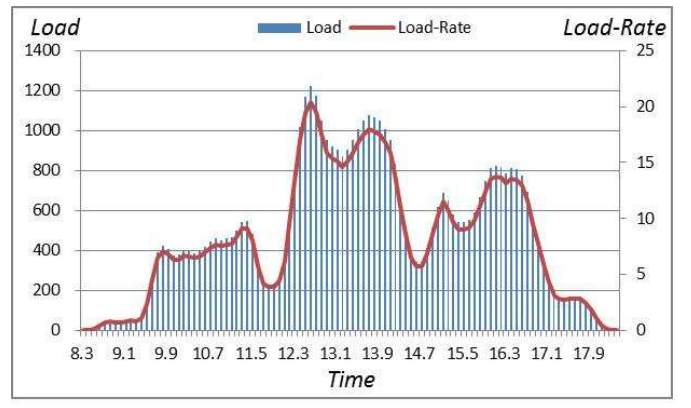

Fig. 8: Spot 7's Number of Tourists over Periods

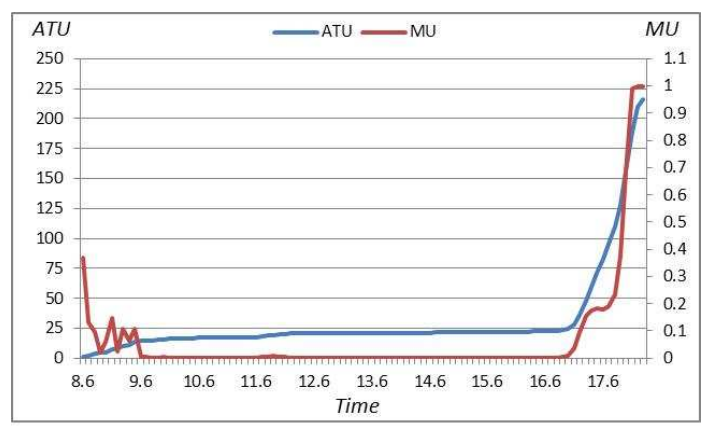

Fig. 9: Accumulated Utility and Marginal Utility of Spot 7

For the spot 7 , the capability $c_{7}=60$ but the actual number of tourists well exceeds 60 , even 1,000 from time to time. And the actual congestion is shown as Figure 7 while the simulation is shown as Figure 8:

This spot is under long-term high-degree congestion from Figure 8, so the marginal utility of tourists tends to be 0 in the long term from Figure 9. Correspondingly, the accumulated utility value increases slowly before 17 o'clock but shoots up due to the decrease of load rate and the increase of marginal utility after 17 o'clock.

According to the above simulation results and actual observation, too small capacity of the spot 7 is the key reason to unbalance of tourists distribution in Jiuzhaigou. Therefore, the management and supervision of this region shall stress this spot and the relevant supervision shall be strengthened on this scenery spot; in addition, it is necessary to expand the capacity of the scenic spot 7 or guide the tourists to other spots in various manners such as price adjustment, vehicle scheduling and information platform.

\section{Conclusion}

Due to unbalanced distribution of tourists during peak time, tourists are likely to highly flock in certain areas of scenery spots if imperfect organization and management.
For this reason, there may be crowds or even trample accidents in addition to unrecoverable damages for the ecological environment in such areas. Therefore, the valid solution is to formulate scientific tourist diversion strategy, reasonably adjust the tour routes, and realize the balanced distribution of tourists over space and time. The prerequisite and base of the research is to establish scientific rational evaluation indicators for diversion strategy. The conventional evaluation models can be only used for macro analysis of the diversion strategies but fail to reveal the inherent problems of each strategy, so they cannot provide scientific, reasonable and specific suggestions for scenic region management. For this reason, this thesis established the tourism utility function (TUF) based on the spot congestion degree in order to provide more specific suggestions for meticulous region management while this model is used for not only diversion strategies evaluation but also analysis of existing specific problems in the strategies. However, for the purposes of simplification, this thesis took account of the congestion degree's effects on the tourism utility during the establishment of TUF Model but ignored other factors. Thus, the follow-up research will give comprehensive consideration of these factors, including costs and time consumed by the tourists during their trip, preferences, distance between adjacent spots, and effects of congestion degree on tourists feelings and experience.

\section{Acknowledgement}

This work was supported by Major International Joint Research Program of the National Natural Science Foundation of China (No.71020107027), the National Science Foundation of China under Grant No.71001075, Central University Fund of Sichuan Unversity No.skqy201112 and Doctoral Fund of Ministry of Education of China (No.20110181110034).

\section{References}

[1] Kreg, L., Lindberg, S. M., and George, S. Rethinking Carrying Capacity. RESEARCH NOTES AND REPORTS, 461-464 (1996).

[2] Anna, L., Alan, F. and Philip, G. Revenue Management in Scottish Visitor Attractions.

[3] Yan, L., Xu, X.G. and Zhang, X.P. Analysis to Temporal Characteristics of Tourist Flows on Jiuzhaigou World Natural Heritage, 45, 171-177 (2009).

[4] Li, J.. The Research of Intelligent Service Management on Large Exhibition. Fudan University, (2010).

[5] Li, J. H. and Zhu, D. L. Visitors Coordination Control Methods in Large-Scale Exhibition Base on Multi-Agent. Journal of System Engineering, 25, 499-505 (2010).

[6] Qiu. Y. Q., Ge, P., Ren, P. Y. A Study on Temporal and Spatial Navigation Based on the Load-Balance of Tourists in Jiuzhaigou Valley. Resources Science, 32, 118-123(2010). 
[7] Liu, Z. S. A Study on Temporal and Spatial Navigation Management Theory and Shuttle Bus Dispatching in National Park of China. Sichuan University, (2012)

[8] V. Shi, J. G. Research on Pedestrian Traffic Characteristics in Special Events. The Academic Committee of Beijing University of Technology, (2007).

[9] Weng, G. M. and Yang X. P. A Critical Survey on the Capacity of Tourist Environment Home and Abroad. Journal of Yanshan University (Philosophy and Social Science Edition), 6, 39-43 (2005).

[10] Ge, P., Zheng, W. M. and Xiao, X. H. et al. Simulation study based on the regional space-time load balancing of Jiuzhaigou. Journal of Industrial Engineering and Engineering Management, 27, (2013).

[11] Gao, H. Y. The Western Economics. The Press of China Economy, (1998)

[12] Xiao, Z. D., Yan, Y., Zhao, X. P.. Research on tourism consumption and its utili. Journal of Shanxi Normal University (Natural Science Edition), 29, 112-117 (2001).

[13] Chen, X. Q., Huang, F. C. Research on the probabilistic travel utility model and the strategy of developing tourism in stagnation area. Zhejiang University (Science Edition), 34, 702-708 (2007).

[14] Feng, G. An Entropy-RFID-Based Management Model for Spatiotemporal Separation Navigation during the Peak Tourist Season in Scenic Areas-An Empirical Study Focusing on Jiuzhaigou National Park. Sichuan University, (2010).

[15] Ge, P., Zheng, W. M. and Xiao, X. H. et al. Simulation study based on the regional space-time load balancing of Jiuzhaigou. Journal of Industrial Engineering and Engineering Management, 27, (2013).

[16] Li, J. and Zhu, D. L. Multi-stage Dynamic Coordination and Control Model for Mass Crowds Activities Based on Multi-agent. Chinese Journal of Management Science, 17, 113-119 (2009).

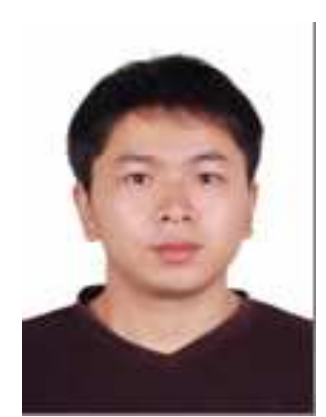

Shaojiang Lin is a doctoral student in Sichuan University for majoring in business management, and a member of Information and Business Management Institute of Sichuan University. His researches mainly relate to strategic management. In recent years, he has published about which are retrieved by EI or CSSCI. ten academic papers, some of

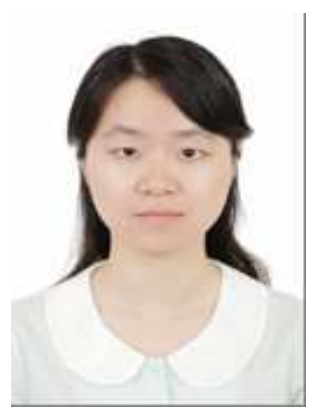

Yuguo Yuan is a graduate student at Sichuan University majoring in Management Science and Engineering. Her researches mainly relate to system simulation and modeling, service operations management and supply chain management, etc. In recent years, she has published several academic papers.

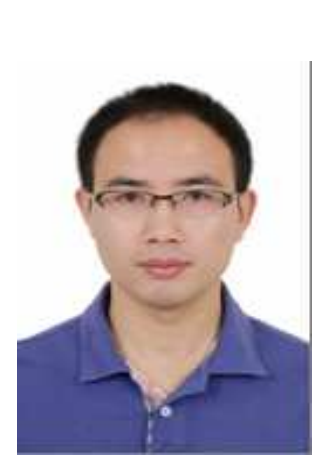

\section{Weimin}

Zheng is a doctoral student at Sichuan University majoring in Management Science and Engineering, and is a member of Information and Business Management Institute of Sichuan University. His researches mainly relate to system simulation and modeling, system and information science and industrial engineering, etc. In recent years, he has published about ten academic papers, some of which are retrieved by SCI, EI or CSSCI. 


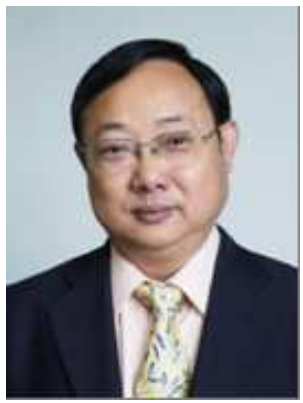

Peiyu Ren was born in Chongqing, China. He is a professor, Ph.D. supervisor. Prof. In recent years, he has presided over and completed five programs of NSFC, one project of State Social Science Found, one key project of Chinese Ministry of Education (Sub-Project), three ministerial and provincial projects and twenty-seven enterprises? projects. At present, he is acting as the director of a large amount of projects and presiding over ?Research on Low-carbon Scenic Integrated Management Model for Harmony Sustainable Development between Tourism Economy and Ecological Environment of Western Scenics? supported by Projects of International Cooperation and Exchanges NSFC(No.71020107027), ?the RFID technology Based on Navigation Management Mode for Spatiotemporal Separation and Its Application in the Nature Conservation Region and Earthquake Relics Museum? supported by National High Technology Research and Development Program of China (No.2008AA04A107) and "Research on Sustainable Development Strategy and Evaluation System in Western Scenics " supported by Doctoral Fund of Ministry of Education of China(No. 20110181110034). In total, he has published 15 monographs and more than one hundred academic papers, including SCI, EI, Management World, China Industrial Economics and so on.

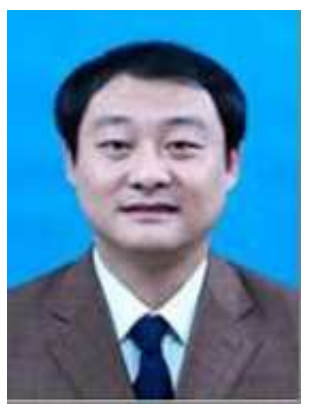

\section{Maozhu}

Jin received his M.S. degree in the Department of Electronics Science and Technology and Ph.D. in the Department of Business from University of Huazhong Science and Technology, Wuhan, China, in 2005 and 2008, respectively. In April 2009, he joined Business School of Sichuan University, Chengdu, China. His research interests include multi-objective optimization, game theory, service science and supply chain management. 